\title{
The scope of Rp EPIC markers in population genetic studies: a preliminary study with dengue vectors
}

\author{
MD Nirmani ${ }^{1}$, NS Perera ${ }^{2}$ and GH Galhena ${ }^{1 *}$ \\ ${ }^{I}$ Department of Zoology and Environmental Science, Faculty of Science, University of Colombo, Colombo 03. \\ ${ }^{2}$ Genetech Molecular Diagnostics and School of Gene Technology, 34, Kitulwatte Road. Colombo 08.
}

\begin{abstract}
Most genetic markers carry numerous limitations which question their credibility. Ribosome protein Exon Primed Intron Crossing (Rp EPIC) markers claimed to effectively overcome some of these limitations. Hence, the present study was aimed at demonstrating the scope of Rp EPIC markers in population genetics in comparison to conventional microsatellites, using the dengue vectors; Aedes aegypti and Aedes albopictus. A total of 62 Ae. aegypti and 51 Ae. albopictus samples were genotyped for two Rp EPIC markers; RpS20b and RpL30a and two microsatellite markers; AC7 and BbH08 for Ae. aegypti and Alb-tri 03 \& Alb-tri 25 for Ae. albopictus. The Rp EPIC markers were successfully transferred among the two species, while differentiating them based on F statistics. Total size variants (electromorphs) observed for the two markers, RpS20b and RpL30a were 14 ( 3 for Ae. aegypti; 13 for Ae. albopictus; shared: 2) and 7 (3 for Ae. aegypti; 4 for Ae. albopictus; shared: 0) with many more alleles uncovered through sequencing (RpS20b:74 and RpL30a:54). In contrast, microsatellites produced 6 (AC7) and $3(\mathrm{BbH} 08)$ size variants for Ae. aegypti and 8 (Alb-tri 03) and 7 (Alb-tri 25) for Ae. albopictus. Both marker types detected genetic structure among Ae. albopictus populations while a genetic structure among Ae. aegypti was detected only with microsatellites and not with Rp EPIC size variants. The combined data of microsatellites and Rp EPIC size variants as well as sequence analysis did not support the presence of a genetic structure among the studied Ae. aegypti populations. It is possible that microsatellites tend to inflate genetic differentiation among the studied Ae. aegypti populations, which has been counteracted by the Rp EPIC markers.
\end{abstract}

Keywords: Aedes aegypti, Aedes albopictus, genetic differentiation, microsatellites, population genetics, size homoplasy.

\section{INTRODUCTION}

During the last fifteen years, use of molecular markers played a major role in understanding the genetic diversity and evolution within living organisms revealing polymorphisms at the DNA level. Selecting an appropriate set of genetic markers, which best answer the target questions would be the first and most critical step in any such study. There are several types of genetic markers, which have been used in population genetic studies of insect vectors and other organisms by numerous scientists with both pros and cons in terms of cost, speed, amount of DNA needed, technical labour, degrees of polymorphism, precision of genetic distance estimates, and statistical power of tests. For example, Restriction Fragment Length Polymorphism (RFLP) markers have been used in analysing population genetic aspects of insect vectors such as Ae. aegypti (Yan et al., 1998) and Anopheles gambiae (Romans et al., 1991) which are expensive, time consuming, less polymorphic and require high quality DNA (Francis et al., 2017; Singh et al., 2017). The Amplified Fragment Length Polymorphism (AFLP) technique was found to be more advantageous than RFLP with improved specificity, reproducibility, abundance on the genome, ability to develop without prior knowledge of the sequence and flexibility to use on both dead and live specimens (Gupta \& Preet, 2014; Singh et al., 2017). However, they are expensive, more laborious, time consuming and are of dominant nature ( Yan et al., 1999; Francis et al., 2017).

\footnotetext{
* Corresponding author (gayanihg@gmail.com; (10 https://orcid.org/0000-0001-5445-8349)
} 
In late 1900's, the development of Polymerase Chain Reaction (PCR) technique led to amazing advances, mainly in research of genetic diversity. Randomly Amplified Polymorphic DNA (RAPD) was the first PCR based technique which was simpler to use, easy to develop and capable of capturing high resolution of diversity across large regions of the genome at one time, but the lack of reproducibility between different runs due to short primer length and dominant nature affected its credibility (Apostol et al., 1996; Francis et al., 2017). With the advent of sequencing techniques, mitochondrial DNA (mtDNA) became the marker of choice for population genetic studies of various organisms including insect vectors such as dengue and malaria species (Yang et al., 2011; Twerdochlib et al., 2012; Tchouassi et al., 2014; Weeraratne et al., 2018; Motoki et al., 2019). Use of this marker in population genetics seemed to be advantageous as they were easy to amplify, sequence and analyse, maternally inherited, and high in evolutionary rate with low effective population size although it carried some limitations such as integration with the nuclear genome, and non-neutral evolution etc. (Hurst \& Jiggins, 2005; Francis et al., 2017). Even the most widely used genetic marker type in the last decade, simple-sequence repeats (SSR, also known as microsatellites) have shown to carry several limitations when used alone; viz. low genomic frequency, null alleles, band stuttering and low polymorphisms (Fagerberg et al., 2001; Janderson et al., 2002; Goubert et al., 2016; Wei et al., 2019). Besides these pros and cons, these markers have been used in population genetic studies covering a large spectrum of fauna and flora in Sri Lanka (Gunawickrama, 2007; Dammannagoda et al., 2008; De Croos \& Pálsson, 2010; Rajkumar et al., 2011; Abeysinghe et al., 2014; Dangalle et al., 2015; Sandamal et al., 2018; Weeraratne et al., 2018). The Ribosomal protein Exon Primed Intron Crossing (Rp EPIC) markers, which have been used to infer genetic variability since 1990's, flank the exon regions and detect the polymorphisms across the conserved intron region (Lessa, 1992; Slade et al., 1993). Although they were primarily used to analyse the natural history of species, more recent studies have proved their usefulness in detecting population genetic structures. Most importantly, they have shown to be transferable among species with the use of same PCR primers and even with the same PCR profiles (White et al., 2015). They were used in population genetic studies of many organisms across various phyla. For example, Touriya et al. (2003) successfully used Rp EPIC markers in population genetic studies in marine and freshwater fish species and were able to assess the universality of primers by testing them on both reptiles (Moorish gecko) and mammals (human and camel). Effectiveness of $\mathrm{Rp}$ EPIC markers with invertebrates was demonstrated in a population genetic and phylogeographical study of six ant species in Brazil (Ströher et al., 2013). Rp EPIC markers have also been successfully used for pests, i.e. to detect the population substructure at temporal and spatial scales in cotton bollworm Helicoverpa armigera in India, Australia and China (Tay et al., 2008) and for disease vectors, i.e. to differentiate dengue vector Ae. aegypti from Australia, parts of Thailand and Vietnam (Endersby et al., 2009; Endersby et al., 2011). It has also been shown that Rp EPIC markers are transferable among related vector species, i.e. same Rp EPIC markers can be used to detect the population structure of Ae. aegypti, Ae. albopictus and Ae. notoscriptus indicating its versatility in vector genetics (White et al., 2015).

However, there are no reports available on studies conducted using Rp EPIC markers in Sri Lankan context. Hence, the aim of the present study was to examine the scope and usefulness of Rp EPIC markers in population genetic studies using dengue vector mosquitoes in Sri Lanka. The rationale for selecting dengue vectors to study the scope of Rp EPIC markers in population genetics is explained below in the context of dengue infection in Sri Lanka.

Sri Lanka is a country which had invested immensely in its struggle to combat dengue outbreaks, since its hyper-endemic situations in 2004 with 15,463 suspected dengue cases and 88 deaths (National Plan of Action for Prevention and Control of Dengue Fever 2005-2009). In 2017, the number of dengue cases reported in the country was 186,101 while the death toll was more than 400 as per the records from the National Dengue Control Unit (NDCU), which is the highest seen in recent years. It is nearly a 12-fold increase in dengue cases compared to the first dengue epidemic in the country in 2004. Hence, dengue infection has become a major contributing factor that declines the health index in Sri Lanka, which necessitates immediate attention by all the respective parties in order to achieve an effective disease control method. Although the World Health Organization (WHO) has endorsed the new dengue vaccine, Dengvaxia, which is anticipated to be $70 \%$ effective, to be used in the countries that are battling with dengue, the clinical trials are still continuing including in Sri Lanka. However, the feasibility of using the vaccine in controlling the dengue transmission is still unclear. Hence, vector elimination still remains as the major control and preventive method of dengue infection in countries batting with dengue epidemics. Although the massive vector control campaigns conducted by various governmental authorities soon after epidemic situations had been successful in reducing the number of dengue cases time-to-time for short periods, the efforts towards a 
consistent dengue control have failed so far. This failure may have been caused by many factors such as genetic changes in the virus, environmental and climatic changes and microevolutionary changes in the mosquito vectors, Ae. aegypti and Ae. albopictus responsible for dengue transmission in Sri Lanka. The selection of dengue vectors to test the scope of Rp EPIC markers thus has focused on the necessity of identifying a more reliable set of genetic markers to uncover the hidden population and genetic parameters of the vector populations in Sri Lanka, aligning with the WHO recommended global strategy of incorporating population and evolutionary genetic information about vectors (WHO, 1999) in designing efficient vector control measures. As a preliminary study, two Rp EPIC markers were analysed; RpS20b and $\mathrm{RpL30a}$ to generate a range of evolutionary genetic information in four populations; two per each species, Ae. aegypti and Ae. albopictus through both allelic size variants and sequencing data. The two Rp EPIC markers were selected based on their reported amplification success, allele polymorphism and the conformity to the Hardy Weinberg equilibrium in both species as shown by Endersby et al. (2009) and White et al. (2015). Further, both markers produced PCR amplicons within the size range that can be separated on polyacrylamide gels which was an added advantage.

The results were compared with the data generated with two comparable microsatellite markers for each species and also with those data generated by combining both the RpEPIC and microsatellite markers to understand the relative role of Rp EPIC markers in the given set-up. The four microsatellite markers used in the study were AC7 (Slotman et al., 2007) and BbH08 (Chambers et al., 2007) for Ae. aegypti and Alb-tri 03 and Alb-tri 25 (Beebe et al., 2013) for Ae. albopictus. The two microsatellite markers, AC7 and BbH08 have been utilised for many population genetic studies of Ae aegypti in the world. For example, AC7 has been used in investigating the population structure of Ae. aegypti in Thailand and Kenya (Slotman et al., 2007) and mainland Southeast Asian countries (Hlaing et al., 2010) whereas BbH08 has been used in Trinidad (Chambers et al., 2007) and Thailand (Olanratmanee et al., 2013). In general, these markers have provided additional tools to understand the population genetic structure and gene flow within $A e$. aegypti populations facilitating the identification of the patterns of disease transmission (Slotman et al., 2007; Hlaing et al., 2010; Olanratmanee et al., 2013). The two microsatellites that amplified Ae. albopictus in this study, Alb-tri 03 and Alb-tri 25 have been first used in a population genetic study conducted in the Australasian region and were found to carry sufficient diversity to detect population differentiation among Ae. albopictus populations (Beebe et al., 2013). Irrespective of the inherent drawbacks of microsatellites, these two markers have proved to be effective in population genetics in many instances (Beebe et al., 2013; Minard et al., 2018).

\section{METHODOLOGY}

Ethical clearance for this study, including the sampling of mosquitoes, was granted by Ethical Review Committee of Institute of Biology, Sri Lanka (ERC IOBSL 12204 15).

\section{Sample collection}

Mosquito samples were collected from areas where each species showed a high prevalence at the time of collection (May - October 2015) based on the information gathered from the Dengue Epidemiology Unit, Ministry of Health. In each study area, an average of 100 ovitraps (ranging from 90 to 110) was placed covering an area of $500 \mathrm{~m}$ in diameter approximately. Although the initial aim was to collect both species from the same sampling areas, this was not possible as adequate numbers of samples of both species could not be collected at the same site. For example, Ae. aegypti was sampled from two urban areas (Colombo city area; number of samples, $\mathrm{N}=32$ ) and Galle Fort; $\mathrm{N}=30$ ). However, the number of $\mathrm{Ae}$. albopictus larvae collected from Colombo and Galle Fort was not sufficient (14 and 11, respectively) for a population genetic study. Accordingly, Ae. albopictus was sampled from two suburban areas due to its reputation to prevail more in suburban and rural areas: Kalamulla $(\mathrm{N}=32)$ in Kalutara District and Imbulgoda $(\mathrm{N}=19)$ in Gampaha District. All four study areas suffer frequent dengue outbreaks due to many reasons. Colombo being the commercial capital of Sri Lanka, encompasses the central hubs of public transportation and all sorts of working places, factories, schools, and shopping malls within the city limits, which cause thousands of private vehicles to enter the city every day from all parts of the country. This endless public and private transportation mainly acts as a good source of vector transfer in and out of the city. Moreover, rapid urbanisation along with poor city sanitation, inappropriate waste disposal and failure to conduct continuous vector removal make home for mosquito breeding. Galle is another urbanised busy city where tourism and fishing industry prevail. Due to the flow of visitors on regular basis coupled with a poor garbage removal system in place, the area provides opportunity to create plenty of mosquito breeding sites and transfer of mosquitoes. On the other hand, Kalamulla and Imbulgoda are residential areas with ample vegetation including home gardens, paddy fields etc. and are located close to towns and commercial centres. 
All the ovitraps used for mosquito collection were prepared according to WHO guidelines (WHO, 2009). Each ovitrap consisted of a $250 \mathrm{~mL}$ black painted plastic container of $6.5 \mathrm{~cm}$ in diameter and $12.0 \mathrm{~cm}$ in height. A stripe of filter paper $(29.7 \mathrm{~cm} \times 7 \mathrm{~cm})$ was laid around the inner surface of each container and it was filled with hay infusion up to about $6.0 \mathrm{~cm}$ level. Hay infusion was prepared previously by steeping $125 \mathrm{~g}$ of dry rice hay in $120 \mathrm{~L}$ of dechlorinated water for $7 \mathrm{~d}$ in a tightly closed plastic bucket. Ovitraps were placed at indoor and outdoor sites in houses in the selected areas choosing spots protected from rain, direct sunlight and beyond reach of children and pets. The ovitraps were collected $5 \mathrm{~d}$ after placement and both eggs and larvae were transferred to the laboratory in glass bottles containing the same hay infusion. Ovitrap placements and collections were done after obtaining the informed consent from the occupants.

Once transferred to the laboratory, eggs were kept for hatching and the larvae were reared up to fourth instar level. At the fourth stage, larvae were killed by direct submergence in absolute ethanol and identified to species level using standard taxonomic keys (Amerasinghe, 1995). Identified Ae. aegypti and Ae. albopictus larvae were preserved in absolute ethanol and stored at $-20{ }^{\circ} \mathrm{C}$ prior to DNA extraction and genetic analysis. In order to avoid incidental sampling of closely related individual mosquitoes, one mosquito larva was randomly selected from each positive ovitrap for the DNA extraction.

\section{Extraction of DNA}

Mosquito larvae preserved in absolute alcohol were air dried and transferred to separate $1.5 \mathrm{~mL}$ microcentrifuge tubes. To ensure complete evaporation of ethanol, larvae were again dried in a $55{ }^{\circ} \mathrm{C}$ dry bath for $20 \mathrm{~min}$ and each larva was crushed using a micropestle to make a fine powder. DNA was extracted with Promega Wizard genomic DNA purification kit, following manufacturer's instructions. The resulting DNA pellet was air dried for approximately $30 \mathrm{~min}$ and resuspended in $100 \mu \mathrm{L}$ of 10 mMTris-1 mM EDTA (pH 8.0). All DNA samples were stored at $4{ }^{\circ} \mathrm{C}$ for short term and at $-20{ }^{\circ} \mathrm{C}$ for the long term.

\section{Generation of Rp EPIC markers}

Since this is only a pilot study, which investigates the feasibility of using Rp EPIC markers in the analysis of population genetics in Ae. aegypti and Ae. albopictus mosquito populations in Sri Lanka, only two markers: RpL30a and RpS20b (Endersby et al., 2009) were used.
Polymerase chain reaction (PCR) amplification of the markers were carried out according to Endersby et al. (2009) with some modifications. Primer sequences for the two markers were RpL30a - Forward primer: ATGGTTACCGCCAAGAAACA, Reverse primer: CGGAGAGTCTTCAGGGTCTG; RpS20b - Forward primer: GCGTATYACCACCCGTAAGA, Reverse primer: GCGAGTGCARATCGATGATA. Each PCR reaction was performed in a $25 \mu \mathrm{L}$ volume comprising $25 \mathrm{ng}$ of extracted DNA, 1X PCR amplification buffer, $1.5 \mathrm{mmol} / \mathrm{L} \mathrm{MgCl}_{2}$ (Promega, USA), $0.2 \mathrm{mmol} / \mathrm{L}$ dNTPs (Promega, USA), $0.1 \mu \mathrm{mol} / \mathrm{L}$ forward primer (IDT, USA) and $0.1 \mu \mathrm{mol} / \mathrm{L}$ reverse primer (IDT, USA) for RpS20b, $0.15 \mu \mathrm{mol} / \mathrm{L}$ forward primer (IDT, USA) and $0.15 \mu \mathrm{mol} / \mathrm{L}$ reverse primer (IDT, USA) for RpL30a and $1.75 \mathrm{U}$ of Go Taq DNA Polymerase (Promega, USA). The same PCR cycling conditions were used for both Rp EPIC markers for both species: initial denaturation $\left(5 \mathrm{~min}, 94{ }^{\circ} \mathrm{C}\right), 35$ cycles of denaturation $(30 \mathrm{~s})$ at $94{ }^{\circ} \mathrm{C}$, annealing $(30 \mathrm{~s})$ at $58{ }^{\circ} \mathrm{C}$ and extension $(30 \mathrm{~s})$ at $72{ }^{\circ} \mathrm{C}$, final extension at $72{ }^{\circ} \mathrm{C}(10 \mathrm{~min})$. Fragments derived from PCR were first run on $2 \%$ agarose gels for qualitative analysis and then separated on $6 \%$ denaturing polyacrylamide gels (PAGE) followed by silver staining to determine the zygosity. The alleles were scored manually based on their sizes using known samples, which were confirmed previously through automated Sanger sequencing.

\section{Processing of heterozygous samples}

All heterozygous samples detected from PAGE were purified to obtain the individual alleles by incising the gel before sending them for sequencing. Initially, the two alleles resulting from any given sample were cut apart from the polyacrylamide gel using a sterile surgical blade and put into separate $1.5 \mu \mathrm{L}$ microcentrifuge tubes. A volume of $50 \mu \mathrm{L}$ of 10 mMTris- 1 mM EDTA ( $\mathrm{pH} 8.0$ ) was added to each tube and they were incubated at $37{ }^{\circ} \mathrm{C}$ overnight to elute DNA from the gel. A second PCR amplification was performed using a volume of $3 \mu \mathrm{L}$ from each eluted DNA sample with the same primers and using the same PCR conditions. PCR amplicons were again run on $2 \%$ agarose and $6 \%$ denaturing polyacrylamide gels to confirm the accuracy of allele purification.

\section{Sanger sequencing}

PCR amplicons of all homozygous samples and purified Rp EPIC alleles of the heterozygous samples were sent to Macrogen, South Korea for automated Sanger sequencing. 


\section{Generation of microsatellite markers}

In order to compare the usefulness of Rp EPIC markers with microsatellite markers in determining population structures of Ae. aegypti and Ae. albopictus, two established microsatellite markers for each species were generated; $\mathrm{AC} 7$ and $\mathrm{BbH} 08$ for Ae. aegypti and Alb-tri 03 and Alb-tri 25 for Ae. albopictus. For each marker, the $25 \mu \mathrm{L}$ PCR reaction mix comprised $25 \mathrm{ng}$ template DNA, $0.2 \mathrm{mmol} / \mathrm{L}$ dNTPs (Promega, USA), $2.5 \mu \mathrm{L}$ of 10X Dream Taq PCR amplification buffer and 1.5 Units of Taq polymerase (Thermo Fisher Scientific, USA), $2.5 \mu \mathrm{mol} / \mathrm{L}$ forward primer (IDT, USA) and $2.5 \mu \mathrm{mol} / \mathrm{L}$ reverse primer (IDT, USA).The PCR cycling conditions for the two species were as follows: For Ae. aegypti, denaturation $\left(4 \mathrm{~min}, 94{ }^{\circ} \mathrm{C}\right), 35$ cycles of $94{ }^{\circ} \mathrm{C}(30 \mathrm{~s})$, annealing $(30 \mathrm{~s}) 55{ }^{\circ} \mathrm{C}$ and extension at $72{ }^{\circ} \mathrm{C}(30 \mathrm{~s})$, with final extension at $72{ }^{\circ} \mathrm{C}(10 \mathrm{~min})$ for $\mathrm{AC} 7$ and denaturation $\left(5 \mathrm{~min}, 94{ }^{\circ} \mathrm{C}\right), 35$ cycles of $94{ }^{\circ} \mathrm{C}(1 \mathrm{~min})$, annealing $(1 \mathrm{~min}) 60{ }^{\circ} \mathrm{C}$ and extension at $72{ }^{\circ} \mathrm{C}(2 \mathrm{~min})$, with final extension at $72{ }^{\circ} \mathrm{C}(10 \mathrm{~min})$ for $\mathrm{BbH} 08$. For Ae. albopictus, denaturation $\left(3 \mathrm{~min}, 95{ }^{\circ} \mathrm{C}\right), 30$ cycles of $95{ }^{\circ} \mathrm{C}(30 \mathrm{~s})$, annealing $(30 \mathrm{~s}) 56{ }^{\circ} \mathrm{C}$ and extension at $72{ }^{\circ} \mathrm{C}(30 \mathrm{~s})$, with final extension at $72{ }^{\circ} \mathrm{C}(5 \mathrm{~min})$ for both primers.

\section{Data analysis}

For both Rp EPIC markers, alleles generated using each method, i.e. with polyacrylamide gel electrophoresis (PAGE) and sequencing were analysed separately. For each marker detected in PAGE (both Rp EPIC and microsatellites), allele numbers, allelic richness, Weir and Cockerham's measure of $F_{\text {IS }}$ and overall $F_{\text {ST }}$ estimates were calculated using FSTAT version 2.9.3 (Goudet, 1995). Estimates of observed $\left(H_{\mathrm{O}}\right)$ and expected $\left(H_{\mathrm{E}}\right)$ heterozygosities, conformity to Hardy-Weinberg equilibrium (HWE), the linkage disequilibrium (LD) and population pairwise genetic differentiation $\left(F_{\mathrm{ST}}\right)$ was estimated in Arlequin version 3.01 (Excoffier \& Liscer, 2006). The level of gene flow between the populations within the species was calculated following the formula $N m=1 / 4\left(1 / F_{\mathrm{ST}}-1\right)$ (Slatkin \& Barton, 1989), where $N$ is the effective population size and $m$ is the migration rate. The null alleles and allele dropouts were determined using MICROCHECKER version 2.2.3 (Van Oosterhout et al., 2004).

Following analyses were conducted using the allele sequences generated from Rp EPIC markers. All the sequences generated for each marker and species were assembled together and aligned using BioEdit 7.2.5 (Hall, 1999), following the ClustalW multiple alignment algorithm with default parameters tovisualise single nucleotide polymorphisms (SNPs) and InDels. BLASTN search tool available at https://blast.ncbi.nlm.nih.gov/ Blast.cgi was used to compare the similarity of the generated sequences to reference genomes of Ae. aegypti and Ae. albopictus.

The Rp EPIC marker DNA sequence variability was analysed in terms of the following parameters using DnaSP version 5.10.01 (Librado \& Rozas, 2009): nucleotide diversity ( $\mathrm{Pi}$ and Watterson's $\theta_{\mathrm{w}}$ ), number of haplotypes, haplotype diversity and the average number of nucleotide differences. In addition, InDel polymorphism of each population of Ae. aegypti and Ae. albopictus was calculated in terms of the total number of InDel sites and events analysed, average InDel length, the number of InDel haplotypes, InDel haplotype diversity, InDel diversity and InDel diversity per site under the multiallelic model which considers all InDel events.

To test whether the sequenced fragments of $\mathrm{Rp}$ EPIC markers deviate from the standard neutral model, Tajima's D (TD), Fu and Li's D* (FLD*) (Fu \& Li, 1993) and Fu and Li's F* (FLF*) (Fu \& Li, 1993) were calculated.

\section{RESULTS AND DISCUSSION}

\section{Rp EPIC marker amplification and polymorphism}

The two Rp EPIC markers, RpS20b and RpL30a were amplified successfully in the samples of both Aedes species under the same PCR conditions. This differs to what had been reported by White et al. (2015) where different annealing temperatures had to be used for the amplification of markers among the two species irrespective of the transferability of the markers. However, the results of this study confirmed that these Rp EPIC markers are not only well transferable between the two species but can be amplified together with much ease, a feature that would be immensely convenient in evolutionary genetic studies.

The level of marker polymorphism (as detected by PAGE) differed widely among the two markers as well as among the two species. A lower allele count was found for RpL30a marker in both Ae. aegypti (Colombo: 2; Galle: 3; shared: 2) and Ae. albopictus (Kalamulla: 2; Imbulgoda: 3; shared :1). For RpS20b marker, allele counts remained low for Ae. aegypti (Colombo:3; Galle: 2; shared: 2) while for Ae. albopictus they were quite high (Kalamulla: 11; Imbulgoda: 7; shared :5) (Table 1). 
Table 1: Population genetic parameters for the two Rp EPIC markers for Ae. aegypti and Ae. albopictus

\begin{tabular}{llcccc}
\hline Marker & Parameter & $\begin{array}{c}\text { Ae. aegypti } \\
\text { Colombo } \\
(\mathrm{N}=32)\end{array}$ & $\begin{array}{c}\text { Ae. aegypti } \\
\text { Galle } \\
(\mathrm{N}=30)\end{array}$ & $\begin{array}{c}\text { Ae. albopictus } \\
\text { Kalamulla } \\
(\mathrm{N}=32)\end{array}$ & $\begin{array}{c}\text { Ae. albopictus } \\
\text { Imbulgoda } \\
(\mathrm{N}=19)\end{array}$ \\
\hline RpS20b & $\mathrm{N}_{\mathrm{PAGE}}$ & 3 & 2 & 11 & 7 \\
& $\mathrm{~N}_{\mathrm{SEQ}}$ & 11 & 12 & 31 & 20 \\
& $F_{\mathrm{IS}}$ & 0.350 & 0.188 & 0.834 & 0.717 \\
& $\mathrm{R}$ & 2.993 & 2.000 & 8.736 & 7.000 \\
& $H_{\mathrm{E}}$ & 0.471 & 0.416 & 0.805 & 0.791 \\
& $H_{\mathrm{O}}$ & 0.313 & 0.345 & 0.138 & 0.235 \\
& HWE $P$ & $0.0058^{*}$ & 0.0229 & $0.0000^{*}$ & $0.0000^{*}$ \\
& $\mathrm{~N}_{\mathrm{PAGE}}$ & 2 & 3 & 2 & 3 \\
& $\mathrm{~N}_{\mathrm{SEQ}}$ & 11 & 5 & 25 & 13 \\
& $F_{\mathrm{IS}}$ & 0.077 & 0.367 & 1.000 & 0.477 \\
& $\mathrm{R}$ & 2.000 & 3.000 & 1.784 & 2.997 \\
& $H_{\mathrm{E}}$ & 0.412 & 0.479 & 0.061 & 0.204 \\
& $H_{\mathrm{O}}$ & 0.387 & 0.310 & 0.000 & 0.111 \\
& HWE $P$ & 0.6798 & 0.0643 & 0.0153 & 0.054 \\
\hline
\end{tabular}

$\mathrm{N}_{\mathrm{PAGE}}$ (allele number), $F_{\mathrm{IS}}$ (inbreeding coefficient), R (allelic richness), $H_{\mathrm{E}}$ (expected heterozygosity), $H_{\mathrm{O}}$ (observed heterozygosity) and HWE $P$ (Hardy-Weinberg Equilibrium p value; * denotes significance after sequential Bonferroni correction) were calculated using Rp EPIC size variants generated on PAGE whereas $\mathrm{N}_{\mathrm{SEQ}}$ was the number of alleles based on Rp EPIC nucleotide sequence data.

For both markers, allele sharing was observed between the populations of the two species (shared alleles for $A e$. aegypti: 2 for each marker; Ae. albopictus: 5 and 1 for RpS20b and RpL30a, respectively). In addition, due to the transferability of the Rp EPIC markers across species, 2 of the alleles accounting for $14.3 \%$ of the total alleles of RpS20b marker were shared by the two species as well. As such, a total of 14 variant alleles were observed for the RpS20b marker (13 alleles from Ae. albopictus, 3 alleles from Ae. aegypti and 2 alleles shared between the species). However, no shared alleles were observed for RpL30a marker (total number of variant alleles: 7). When the number of alleles observed in this study is compared with the reports from elsewhere, it showed that Sri Lankan Ae. albopictus populations are much more diverse with respect to RpS20b marker compared to those found in Spain and Indonesia (3 alleles each) (White et al., 2015). However, Ae. aegypti populations of the current study showed 3 alleles for the same marker compared to a similar study where 6 alleles had been reported for Ae. aegypti populations collected from three countries: Australia and some parts of Thailand and Vietnam (Endersby et al., 2009). Given the fact that larger countries harbour higher genetic diversity in general, this observation is suggestive of a relatively larger genetic diversity for the Sri Lankan mosquito population in relation to its small geographical size compared to much bigger countries. Similarly, there are other reports of comparable or lower allelic variation for the studied Rp EPIC markers ( 2 alleles each) in both field caught and laboratory reared strains of Ae. aegypti (Yeap et al., 2011). Further, the observed difference in allelic diversity between local Ae. aegypti and Ae. albopictus populations might be stemming from the rapid growth and distribution of Ae. albopictus populations as a result of its high invasive nature and successful adaptability to the environmental changes (Bonizzoni et al., 2013; Waldock et al., 2013).

When the two markers were tested for the conformity to Hardy-Weinberg equilibrium (HWE), three of the eight tests showed deviations from the HWE: RpS20b for Ae. albopictus from Kalamulla and Imbulgoda and RpL30a for Ae. albopictus from Kalamulla (Table 1). Since analysis with MICROCHECKER indicated the presence of null alleles in the respective populations, the observed deviations from HWE may have resulted from these null allele problems. However, there were no large allele dropouts and linkage disequilibrium $(\mathrm{p}>0.05$ for both populations together and separately) with respect to the studied markers and populations. 
Inbreeding coefficients detected for those populations which did not confer to HWE were relatively high and ranged from 0.350 (Ae. aegypti in Colombo for RpS20b) to 1.000 (Ae. albopictus in Kalamulla for RpL30a) (Table 1). It is interesting to note that all Ae. albopictus individuals collected from Kalamulla were homozygous for RpL30a marker with only two alleles, resulting in the maximum possible inbreeding coefficient. A plausible explanation for this observed homozygosity of RpL30a marker is allele fixation driven by the genetic drift. However, the substantial heterozygosity levels observed for the other marker suggests a population at mutation drift equilibrium rendering this assumption unlikely. In addition, RpL30a marker showed substantial heterozygosity in the other three populations tested in the present study as well as in previous research conducted elsewhere (Endersby et al., 2009, 2011; Olanratmanee et al., 2013; White et al., 2015). This excludes the possibility of a function related sequence conservation or genetic hitchhiking for the RpL30a marker. Thus, it is likely that the observed homozygosity has been caused by the limited sample number in the current study, which necessitates further analysis of the population with a larger sample size.

\section{Rp EPIC marker-based population differentiation}

Pairwise $F_{\mathrm{ST}}$ estimates obtained for the two populations within each species were substantially low and were not statistically significant $(\mathrm{p}>0.05)$ indicating lack of population structure among the studied areas (Table 2, 'below diagonal without parenthesis'). Further, as expected, when populations between the two species were compared, there was significant differentiation $(\mathrm{p}<0.05)$ among all respective populations indicating the high discriminatory capacity of these markers notwithstanding the transferability of the markers across species. The overall estimates of $\mathrm{Nm}$ between populations within species were higher $(\infty)$ for Ae. aegypti compared to Ae. albopictus (3.08) denoting a higher level of mosquito migration within the two Ae. aegypti populations despite their distant geographical locations.

Table 2: $\quad$ Population pairwise measures of $F_{\mathrm{ST}}$ for both Aedes species, generated with Rp EPIC markers, microsatellite markers and combining Rp EPIC and microsatellite markers

\begin{tabular}{|c|c|c|c|c|}
\hline & $\begin{array}{l}\text { Ae. aegypti } \\
\text { Colombo }\end{array}$ & $\begin{array}{l}\text { Ae. aegypti } \\
\text { Galle }\end{array}$ & $\begin{array}{l}\text { Ae. albopictus } \\
\text { Kalamulla }\end{array}$ & $\begin{array}{l}\text { Ae. albopictus } \\
\text { Imbulgoda }\end{array}$ \\
\hline $\begin{array}{l}\text { Ae. aegypti } \\
\text { Colombo }\end{array}$ & 0.0000 & 0.0232 & 0.2919 & 0.2841 \\
\hline $\begin{array}{l}\text { Ae. aegypti } \\
\text { Galle }\end{array}$ & $\begin{array}{l}0.0089 \\
(-0.0044)\end{array}$ & 0.0000 & 0.1883 & 0.1719 \\
\hline $\begin{array}{l}\text { Ae. albopictus } \\
\text { Kalamulla }\end{array}$ & $\begin{array}{l}0.7655 \\
(0.7655)\end{array}$ & $\begin{array}{l}0.7392 \\
(0.7460)\end{array}$ & 0.0000 & -0.0035 \\
\hline $\begin{array}{l}\text { Ae. albopictus } \\
\text { Imbulgoda }\end{array}$ & $\begin{array}{l}0.6640 \\
(0.6574)\end{array}$ & $\begin{array}{l}0.6277 \\
(0.6300)\end{array}$ & $\begin{array}{l}0.0658 \\
(0.0899)\end{array}$ & 0.0000 \\
\hline
\end{tabular}

Above the diagonal: $F_{\mathrm{ST}}$ estimates calculated by microsatellite markers; below the diagonal: $F_{\mathrm{ST}}$ estimated with Rp EPIC size variants $\left(F_{\mathrm{ST}}\right.$ estimated with combined EPIC and microsatellite markers). Bold denotes significance at the table-wide $\alpha=0.05$ level.

\section{Rp EPIC marker sequence analysis}

In addition to analysing Rp EPIC markers based on polyacrylamide genotyping, all the resolved fragments were sequenced to identify the underlying sequence heterogeneity. Sequences of both markers (RpS20b and RpL30a) were compared with GenBank reference sequences and all showed $>93 \%$ identity with a sequence coverage of $100 \%$. Further, all sequences of Ae. aegypti showed highly significant hits to the Ae. albopictus genome, and vice-versa, with low sequence coverage (54\% - $70 \%$ ) but high sequence identity (> $84 \%$ ), reflecting the observed marker transferability between the two species.

\section{Rp EPIC marker nucleotide variation}

For the two EPIC markers, the average number of bases analysed was 480 for Ae. aegypti and 464 for Ae. albopictus. Out of $399 \mathrm{Rp}$ EPIC nucleotide 
sequences generated, only $85.5 \%$ was successful, mostly due to 1-2 base pair differences present in heterozygous target alleles, which render the gel purification of alleles difficult. Sequencing confirmed the allele sizes obtained through polyacrylamide gels for most samples except a few, especially in Ae. albopictus populations for RpS20b marker, probably due to the low separation of the two heterozygous alleles caused by a 1 or 2 base pair difference. Nevertheless, sequencing of Rp EPIC alleles was able to resolve the majority of observed allelic variations with some minor discrepancies in allele sizes estimated from polyacrylamide gels, which highlight the importance of sequencing in population genetic studies. In addition to the allelic variability found on polyacrylamide gels, sequencing revealed an ample number of polymorphic sites for all four populations, which can be used as additional reproducible markers for evolutionary genetic studies. As such, sequences of 38 alleles [30 size variants +8 single nucleotide polymorphisms (SNPs)] were deposited in NCBI GenBank Repository (Accession numbers KU682356KU682357, KU682769-KU682770, KX364713, KY319144-KY319167, KY586244-KY586251 and KY883999).

\section{Size homoplasy in Rp EPIC markers}

For both species, sequence analysis yielded a higher allele number in comparison to the alleles scored through PAGE, exhibiting an extensive amount of size homoplasy. The highest difference was marked for Ae. albopictus from Kalamulla population for RpL30a marker, which was a 12.5-fold increase of allelic variation in comparison to allele scoring on PAGE. The lowest difference (3) was shown for Ae. aegypti from Galle for the same marker (Table 1). In general, the average allele numbers generated from sequence analysis per marker and population were 9.75 and 22.25 for $\mathrm{Ae}$. aegypti and Ae. albopictus, respectively which were significantly higher $(\mathrm{p}<0.05)$ than the allele numbers obtained via PAGE. This indicates that sequencing of Rp EPIC markers significantly increased the level of marker polymorphism compared to the results obtained through electromorphs detected on PAGE. This increased amount of polymorphism could be further utilised as separate genetic markers in population and evolutionary studies, which is an added advantage of using Rp EPIC markers. However, in more conventional size variants like microsatellites, the number of sequence variants are observed to be lesser (Viard et al., 1998).

\section{Nucleotide and haplotype diversity of Rp EPIC alleles}

When nucleotide diversity $(\mathrm{Pi})$ values were calculated, Ae. albopictus samples showed the largest values for both markers; 0.0654 (Imbulgoda population) for $\mathrm{RpS} 20 \mathrm{~b}$ and 0.0249 (Kalamulla) for RpL30a in comparison to Ae. aegypti. Within the populations of each species, Pi averaged over the two Rp EPIC markers did not differ much; for Ae. aegypti $\mathrm{Pi}$ for Galle and Colombo

Table 3: Nucleotide and haplotype diversity of Ae. aegypti and Ae. albopictus populations estimated using Rp EPIC marker sequence data

\begin{tabular}{lccccc}
\hline Marker name & Parameter & $\begin{array}{c}\text { Ae. aegypti } \\
\text { Colombo }\end{array}$ & $\begin{array}{c}\text { Ae. aegypti } \\
\text { Galle }\end{array}$ & $\begin{array}{c}\text { Ae. albopictus } \\
\text { Kalamulla }\end{array}$ & $\begin{array}{c}\text { Ae. albopictus } \\
\text { Imbulgoda }\end{array}$ \\
\hline RpS20b & $\mathrm{Pi}$ & 0.0158 & 0.0161 & 0.0334 & 0.0654 \\
& $\theta$ & 0.0112 & 0.0128 & 0.0389 & 0.0966 \\
& $\mathrm{H}$ & $11(3.41)$ & $11(3.40)$ & $30(9.62)$ & $19(6.09)$ \\
& $\mathrm{Hd}$ & 0.671 & 0.727 & 0.992 & 0.995 \\
$\mathrm{KpL30a}$ & $\mathrm{k}$ & 4.959 & 4.021 & 9.508 & 18.305 \\
& $\mathrm{Pi}$ & 0.0161 & 0.0178 & 0.0249 & 0.0093 \\
& $\theta$ & 0.0213 & 0.0142 & 0.0397 & 0.0138 \\
& $\mathrm{H}$ & $10(6.37)$ & $5(3.18)$ & $21(13.82)$ & $9(5.88)$ \\
& $\mathrm{Hd}$ & 0.610 & 0.693 & 0.942 & 0.724 \\
& $\mathrm{k}$ & 2.461 & 2.736 & 3.742 & 1.314 \\
\hline
\end{tabular}

Pi: nucleotide diversity (the average number of nucleotide differences per site between two sequences), $\theta$ : nucleotide diversity (nucleotide diversity calculated using the number of segregating sites), H: number of haplotypes based on DNA polymorphism exclusive of InDels (percent number of haplotypes to the total number of sites analyzed), Hd: DNA polymorphism haplotype diversity and $\mathrm{k}$ : average number of nucleotide differences. 
populations were 0.01695 and 0.01595 , respectively while for Ae. albopictus, Pi for Imbulgoda and Kalamulla populations were 0.0435 and 0.0304 , respectively. The same pattern was observed for nucleotide diversity $(\theta)$, which estimates the population mutation rate from number of polymorphic sites. The haplotype diversity averaged over markers (Hd) were comparable for the two populations within each species (for Ae. aegypti, $\mathrm{Hd}_{\text {Colombo }}: 0.6405 ; \mathrm{Hd}_{\text {Galle }}: 0.71$ and for Ae. albopictus, $\left.\mathrm{Hd}_{\text {Kalamulla }}: 0.966 ; \mathrm{Hd}_{\text {Imbulgoda }}: 0.8595\right)$, although in between species, it was significantly higher for Ae. albopictus (0.9133) compared to Ae. aegypti (0.6753) (Table 3). The $\mathrm{Pi}$ values obtained for Ae. aegypti populations for both markers of this study were comparable to the $\mathrm{Pi}$ values that have been previously reported for $\mathrm{Ae}$. aegypti from Queensland and Brazil (White et al., 2015). Ae. albopictus revealed moderately higher or similar diversity levels to Spain and Indonesian populations for RpS20b but lower diversity values with respect to RpL30a marker. As a whole, the two local Ae. albopictus populations seemed to be more diverse than the two local Ae. aegypti populations.

\section{InDel (Insertion-Deletion) polymorphism}

In general, the number of InDel sites was highest for Ae. albopictus from Imbulgoda for both Rp EPIC markers (RpS20b:21; RpL30a:12). For individual populations however, average InDel diversity across both markers did not differ significantly from each other in both species; i.e., for Ae. aegypti - 1.095 for Colombo and 1.004 for Galle: for Ae. albopictus - 1.0125 for Kalamulla and 0.967 Imbulgoda. The InDel length averaged over the two markers were $2.25 \mathrm{bp}$ for Ae. aegypti and $1.55 \mathrm{bp}$ for Ae. albopictus. However, the mean InDel diversity across markers did not significantly differ $(\mathrm{p}>0.05)$ between the species i.e., Ae. aegypti:1.0495 or $1 \mathrm{InDel}$ per $36 \mathrm{bp}$; Ae. albopictus: 0.9898 or 1 InDel per $21 \mathrm{bp}$. Nevertheless, these values are 5-6 times larger than the values reported in a study done by White et al. (2015) (i.e. 1 InDel per $144 \mathrm{bp}$ and $198 \mathrm{bp}$ for Ae. albopictus and Ae. aegypti, respectively), which indicates an increased genetic diversity reported by the local dengue vectors (Table 4)

Table 4: InDel (Insertion-Deletion) polymorphism of Ae. aegypti and Ae. albopictus populations estimated using Rp EPIC marker sequence data

\begin{tabular}{llcccc}
\hline Marker name & Parameter & $\begin{array}{c}\text { Ae. aegypti } \\
\text { Colombo }\end{array}$ & $\begin{array}{c}\text { Ae. aegypti } \\
\text { Galle }\end{array}$ & $\begin{array}{c}\text { Ae. albopictus } \\
\text { Kalamulla }\end{array}$ & $\begin{array}{c}\text { Ae. albopictus } \\
\text { Imbulgoda }\end{array}$ \\
\hline RpS20b & $\mathrm{N}_{\text {Insite }}$ & $9(2.79)$ & $10(3.09)$ & $9(2.88)$ & $21(6.73)$ \\
& $\mathrm{N}_{\text {Inevent }}$ & $4(1.24)$ & $5(1.54)$ & $9(2.88)$ & $14(4.49)$ \\
& $\mathrm{L}_{\text {In }}$ & 2.379 & 2.674 & 1.000 & 1.212 \\
& $\mathrm{H}_{\text {In }}$ & $3(0.93)$ & $4(1.24)$ & $13(4.17)$ & $9(2.88)$ \\
& $\mathrm{Hd} \mathrm{d}_{\text {In }}$ & 0.506 & 0.487 & 0.879 & 0.705 \\
& $\mathrm{k}(\mathrm{i})$ & 1.512 & 1.393 & 1.962 & 1.658 \\
& $\mathrm{Pi}(\mathrm{i})$ & 0.0047 & 0.0043 & 0.0067 & 0.0055 \\
& $\mathrm{~N}_{\text {Insite }}$ & $4(2.55)$ & $3(1.91)$ & $2(1.28)$ & $12(7.84)$ \\
& $\mathrm{N}_{\text {Inevent }}$ & $2(1.27)$ & $1(0.64)$ & $1(0.64)$ & $2(1.31)$ \\
& $\mathrm{L}_{\text {In }}$ & 2.000 & 1.917 & 2.000 & 1.909 \\
& $\mathrm{H}_{\text {In }}$ & $3(1.91)$ & $3(1.91)$ & $2(1.28)$ & $3(1.96)$ \\
& $\mathrm{Hd}$ In & 0.567 & 0.567 & 0.063 & 0.267 \\
& $\mathrm{k}(\mathrm{i})$ & 0.678 & 0.615 & 0.063 & 0.276 \\
& $\mathrm{Pi}(\mathrm{i})$ & 0.0043 & 0.0039 & 0.0004 & 0.0018 \\
\hline
\end{tabular}

$\mathrm{N}_{\text {Insite }}$ : total number of InDel sites analysed (percent total number of InDel sites to the total number of sites analyzed), $\mathrm{N}_{\text {Inevent }}$ : total number of InDel events analysed (percent total number of InDel events to the total number of sites analyzed), $\mathrm{L}_{\mathrm{In}}$ : average InDel length, $\mathrm{H}_{\mathrm{In}}$ : number of InDel haplotypes (percent number of InDel haplotypes to the total number of sites), $\mathrm{Hd}_{\mathrm{In}}$ : InDel haplotype diversity, k(i): InDel diversity, Pi(i): InDel diversity per site. 
Table 5: Statistics of neutrality of Ae. aegypti and Ae. albopictus populations estimated using Rp EPIC marker sequence data

\begin{tabular}{lccccc}
\hline Marker name & Parameter & $\begin{array}{c}\text { Ae. aegypti } \\
\text { Colombo }\end{array}$ & $\begin{array}{c}\text { Ae. aegypti } \\
\text { Galle }\end{array}$ & $\begin{array}{c}\text { Ae. albopictus } \\
\text { Kalamulla }\end{array}$ & $\begin{array}{c}\text { Ae. albopictus } \\
\text { Imbulgoda }\end{array}$ \\
\hline RpS20b & TD & 1.315 & 0.838 & -0.948 & -1.793 \\
& FLD & 1.127 & -0.339 & -0.533 & $-2.558^{*}$ \\
& FLF & 1.403 & 0.058 & -0.796 & $-2.716^{*}$ \\
RpL30a & TD & -0.929 & 0.810 & -1.484 & -1.052 \\
& FLD & -2.376 & -0.408 & -2.137 & 0.028 \\
& FLF & -2.240 & -0.064 & -2.268 & -0.325 \\
\hline
\end{tabular}

TD: Tajima's D; FLD: Fu and Li’s D; FLF: Fu and Li's F; FLF. * denotes $p<0.05$

\section{Haplotype analysis}

When the Rp EPIC sequences were analysed for the haplotypes, a substantial diversity was observed for the two species. The highest number of haplotypes was observed for Ae. albopictus (total of 47; Kalamulla: 29, Imbulgoda:18) for the RpS20b marker whereas the lowest was reported for Ae. aegypti (14; Colombo: 10, Galle: 6, Shared: 2) for the RpL30a marker. Among these, the majority were singleton haplotypes (42 and 25 haplotypes in Ae. albopictus and 12 and 11 haplotypes in Ae. aegypti, for RpS20b and RpL30a, respectively). The number of common haplotypes remained low (2-5 haplotypes) in both species, while a large number of private haplotypes [ranging from 29 (Ae. albopictus from Kalamulla for RpS20b) to 4 (Ae. aegypti from Galle for RpL30a)] were observed. The shared haplotypes were more common in Ae. aegypti [RpS20b: 5 (26.3\%), RpL30a: 2 (14.3\%)] in comparison to Ae. albopictus [RpS20b: 0, RpL30a: 4 (12.9 \%)]. This indicates an elevated level in exchange of genetic material among Ae. aegypti compared to Ae. albopictus, which was further supported by geneflow estimates of the study.

\section{Statistics of neutrality based on Rp EPIC markers}

The neutral evolution of the two markers was tested with Tajima's D for the two species. For Ae. aegypti, Tajima's D showed positive values compared to the negative values obtained for Ae. albopictus. However, none of the values obtained were significantly different from zero for both species. Nevertheless, Ae. albopictus from Imbulgoda showed significantly negative values for $\mathrm{Fu}$ and $\mathrm{Li}^{\prime} \mathrm{D}^{*}$ test $(-2.729)$ and $\mathrm{Fu}$ and $\mathrm{Li}^{\prime} \mathrm{F}^{*}$ test (-2.723) for RpS20b marker (Table 5), reflecting a possible population expansion within that population. This is further evidenced by the high haplotype diversities observed for Ae. albopictus population at Imbulgoda with 16 singleton haplotypes without any common haplotypes for the same RpS20b marker suggesting that this population was maintaining most of the new mutations while expanding with respect to that marker. This observation is consistent with the fact that Ae. albopictus is the most invasive mosquito species in the world showing an immense capacity to adapt.

\section{Comparison of allelic data between microsatellites and Rp EPIC markers}

The analysis on HWE showed that only five comparisons out of eight conferred to HWE $(p<0.05)$ for the Rp EPIC markers, whereas for the microsatellites, all eight comparisons were in HWE $(p<0.05)$. However, no significant linkage disequilibrium was found $(\mathrm{p}>0.05)$ for both the marker types. MICROCHECKER could find null alleles for the two Ae. albopictus populations for both Rp EPIC and microsatellite markers but not in Ae. aegypti populations for either of the markers. Nevertheless, both species did not show large allele dropouts for either Rp EPIC or microsatellite marker types. These results reflect the general suitability of both the marker types in evolutionary studies.

The number of size variants shown by microsatellites for the two species were $6(\mathrm{AC} 7)$ and $3(\mathrm{BbH} 08)$ for Ae. aegypti and 8 (Alb-tri 03) and 7 (Alb-tri 25) for Ae. albopictus. When the genetic diversity indices are compared among the two marker types, microsatellites tend to exhibit a larger diversity compared to the $\mathrm{Rp}$ EPIC markers, especially for Ae. aegypti (Table 6). For Ae. albopictus, this difference is much less prominent. This is probably due to the fact that microsatellites only capture certain intronic areas where variability is concentrated, while Rp EPIC markers screen the total 
intron area, including those more conserved functionally significant regions. Nevertheless, the effective number of alleles or the number of equally frequent alleles that would take to achieve a given level of gene diversity in a population, calculated per marker was comparable between the two marker types for both the species (for Rp EPIC markers, Ae. aegypti: 1.829; Ae. albopictus: 3.056 and for microsatellite markers, Ae. aegypti: 2.488; Ae. albopictus: 3.174$)$.

Table 6: A comparison of population genetic parameters obtained for Ae. aegypti and Ae. albopictus for Rp EPIC and microsatellite markers as detected by polyacrylamide gel electrophoresis

\begin{tabular}{lccc}
\hline Species & Parameter & Rp EPIC & Microsatellites \\
\hline Ae. aegypti & $\mathrm{N}_{\mathrm{PAGE}}$ & 2.5 & $3.75^{*}$ \\
& $\mathrm{R}$ & 2.498 & $3.749^{*}$ \\
& $\mathrm{~N}_{\mathrm{E}}$ & 1.829 & 2.488 \\
& $H_{\mathrm{O}}$ & 0.339 & $0.558^{*}$ \\
& $H_{\mathrm{E}}$ & 0.451 & 0.573 \\
Ae. albopictus & $\mathrm{N}_{\mathrm{PAGE}}$ & 5.75 & 5 \\
& $\mathrm{R}$ & 5.129 & 3.508 \\
& $\mathrm{~N}_{\mathrm{E}}$ & 3.056 & 3.174 \\
& $H_{\mathrm{O}}$ & 0.121 & $0.435^{*}$ \\
& $H_{\mathrm{E}}$ & 0.465 & 0.625 \\
\hline
\end{tabular}

$\mathrm{N}_{\mathrm{PAGE}}$ : average allele number per marker and population detected by polyacrylamide gel electrophoresis, R: average allelic richness per marker and population, $\mathrm{N}_{\mathrm{E}}$ : effective number of alleles per marker and population, $H_{\mathrm{O}}$ : average observed heterozygosity per marker and population, $H_{\mathrm{E}}$ : average expected heterozygosity per marker and population, $*$ denotes significance at table-wise $\alpha=0.05$.

Pairwise $F_{\mathrm{ST}}$ measures calculated using microsatellite markers indicated significant population differentiation $(\mathrm{p}<0.05)$ between the two species (Table 2, above diagonal). A similar observation was found in the analysis of Rp EPIC markers (Table 2, below diagonal without parenthesis) as well as in the analysis where the results of both Rp EPIC and microsatellite markers were combined (Table 2, below diagonal within parenthesis). Further, microsatellite markers showed a significant differentiation between the two Ae. aegypti populations - Colombo and Galle (Table 2, above diagonal), which was not reflected in Rp EPIC marker analysis. However, the combined marker analysis showed a significant population differentiation among the two Ae. albopictus populations. Due to the relatively poor agreement shown with respect to the $F_{\mathrm{ST}}$ estimates derived from the two marker types at population level, haplotypes obtained through the Rp EPIC sequence data were investigated to understand the level of population differentiation at a greater depth. Accordingly, a considerable percentage of shared haplotypes [5 out of 19 haplotypes $(26.32 \%)$ for RpS20b and 2 out of 14 (14.29\%) for RpL30a] were found between the two Ae. aegypti populations indicating a substantial amount of genetic relatedness between them. In contrast, only a small percentage of shared haplotypes were visible among the two Ae. albopictus populations [ 0 out of $47(0 \%)$ haplotypes for RpS20b and 4 out of 31 $(12.90 \%)$ for RpL30a]. In addition, a larger number of private haplotypes was also observed for Ae. albopictus. These results question the reliability of microsatellitebased estimates on genetic differentiation, which are likely to be inflated due to its inherent larger variability. With this respect, Rp EPIC - based estimates seem to be far more reliable for two reasons. First that it captures a variability dispersed over a larger gene area which exclude the possible bias of selecting only those highly variable smaller gene segments. Secondly it allows the evolutionary question at hand to be investigated in different angles to come up with more robust inferences, i.e. analysing molecular size variant data together with haplotypes and/or nucleotide diversity, etc.

The same trend was observed when the global $F_{\text {ST }}$ values were calculated for the two marker types. For microsatellite markers, the global $F_{\mathrm{ST}}$ values were higher for both Ae. aegypti (0.022) and Ae. albopictus (0.16) indicating a substantial genetic structure for the two species, as opposed to the global $F_{\mathrm{ST}}$ estimates obtained based on Rp EPIC marker data generated on PAGE without sequencing (Ae. aegypti: -0.002; Ae albopictus: 0.075 ), or $F_{\mathrm{ST}}$ estimates yielded in combined analysis of microsatellite and Rp EPIC marker PAGE-generated data (Ae. aegypti: -0.004; Ae. albopictus: 0.089), which indicate subtle or absence of a genetic structure. The global $F_{\mathrm{ST}}$ values generated based on Rp EPIC marker sequence analysis (Ae aegypti: -0.0015 ; Ae albopictus: 0.0753 ) was also quite similar to the results obtained through PAGE analysis of Rp EPIC markers. These observations question the reliability of marker dependent genetic inferences made on population characteristics. It may probably be more appropriate to combine several marker types with different characteristics, such as the combined analysis of Rp EPIC and microsatellites as demonstrated here, to counteract the inflation brought in by one marker, while allowing its desirable qualities to be utilised nevertheless. 


\section{CONCLUSION}

Rp EPIC markers, RpL30a and RpS20b were successfully utilized in population genetic analysis of the selected Ae. aegypti and Ae. albopictus populations in Sri Lanka. These were transferable among the two-sister species and allowed for successful multiplex amplification. So far, this is the first study in Sri Lanka that has investigated the scope of Rp EPIC markers in molecular genetic studies. As was apparent with the sequencing of size variants, a relatively large amount of polymorphism was found with both Rp EPIC markers, although allelic diversity of RpL30a marker was relatively low compared to RpS20b among the size variants. However, both markers exhibited null alleles in some of the populations analysed. Further, as revealed by the current study, when analysed along with microsatellites, Rp EPIC markers are likely to counteract the inflation of evolutionary genetic parameters. This might have resulted from the relative low variability of Rp EPIC markers, which analyse the entire intronic regions compared to the microsatellites that focus only on selected highly variable regions.

\section{Acknowledgement}

The authors acknowledge the financial support by the University of Colombo, Sri Lanka (Grant number $\mathrm{AP} / 3 / 2 / 2015 / \mathrm{SG} / 30)$.

\section{REFERENCES}

Abeysinghe P.D., Samarajeewa N.G.C.D., Li G. \& Wijesinghe K.G.G. (2014). Preliminary investigation for the identification of Sri Lankan Cinnamomum species using randomly amplified polymorphic DNA (RAPD) and sequence related amplified polymorphic (SRAP) markers. Journal of the National Science Foundation of Sri Lanka 42(3): 201-208.

Amerasinghe F.P. (1995). Illustrated keys to the genera of mosquitoes (Diptera:Culicidae) in Sri Lanka. Journal of the National Science Council of Sri Lanka 23(4): 183-211.

Apostol B.L., Black IV W.C., Reiter P. \& Miller B.R. (1996). Population genetics with RAPD-PCR markers: The breeding structure of Aedes aegypti in Puerto Rico. Heredity 76(4): 325-334.

DOI: https://doi.org/10.1038/hdy.1996.50

Beebe N.W. et al. (12 authors) (2013). Tracing the tiger: population genetics provides valuable insights into the Aedes (Stegomyia) albopictus invasion of the Australasian region. PLoS Neglected Tropical Diseases 7(8): e2361. DOI: https://doi.org/10.1371/journal.pntd.0002361

Bonizzoni M., Gasperi G., Chen X. \& James A.A. (2013). The invasive mosquito species Aedes albopictus: current knowledge and future perspectives. Trends in Parasitology 29(9): 460-468.
DOI: https://doi.org/ 10.1016/j.pt.2013.07.003.

Chambers E.W., Meece J.K., McGowan J.A., Lovin D.D., Hemme R.R., Chadee D.D., McAbee K., Brown S.E., Knudson D.L. \& Severson D.W. (2007). Microsatellite isolation and linkage group identification in the yellow fever mosquito Aedes aegypti. Journal of Heredity 98(3): 202-210.

DOI: https://doi.org/10.1093/jhered/esm015

Dammannagoda S.T., Hurwood D.A. \& Mather P.B. (2008). Evidence for fine geographical scale heterogeneity in gene frequencies in yellowfin tuna (Thunnus albacares) from the north Indian Ocean around Sri Lanka. Fisheries Research 90(1-3): 147-157.

DOI: https://doi.org/10.1016/j.fishres.2007.10.006

Dangalle C.D., Pallewatte N. \& Vogler A.P. (2015). Inferring population history of tiger beetle species of Sri Lanka using mitochondrial DNA sequences. Ceylon Journal of Science (Biological Sciences) 43(2): 47-63.

DOI: https://doi.org/10.4038/cjsbs.v43i2.7324

De Croos M.D.S.T. \& Pálsson S. (2010). Mitochondrial DNA variation and population genetic structure of white shrimp Fenneropenaeus indicus along the coastal belt of Sri Lanka. Aquatic Living Resources 23(3): 315-323. DOI: https://doi.org/10.1051/alr/2010027

Endersby N. et al. (12 authors) (2009). Genetic structure of Aedes aegypti in Austrailia and Vietnam revealed by microsatellite and exon primed intron crossing markers suggests feasibility of local control options. Journal of Medical Entomology 46(5): 074-1083. DOI:https://doi.org/10.1603/033.046.0514

Endersby N.M., Hoffmann A.A., White V., Ritchie S.A., Johnson P.H. \& Weeks A.R. (2011). Changes in the genetic structure of Aedes aegypti (Diptera: Culicidae) populations in Queensland, Australia, across two seasons: implications for potential mosquito releases. Journal of Medical Entomology 48(5): 999-1007.

Excoffier L., Laval G. \& Schneider S. (2007). Arlequin (version 3.0): an integrated software package for population genetics data analysis. Evolutionary Bioinformatics Online 1: 47-50. DOI: https://doi.org/10.1111/j.1755-0998.2010.02847.x

Fagerberg A.J., Fulton R.E., Black IV W.C. (2001). Microsatellite loci are not abundant in all arthropod genomes: Analyses in the hard tick, Ixodes scapularis and the yellow fever mosquito, Aedes aegypti. Insect Molecular Biology 10(3): 225-236.

DOI: https://doi.org/10.1046/j.1365-2583.2001.00260.x

Fu Y.X. \& Li W.H. (1993). Statistical tests of neutrality of mutations. Genetics 133(3): 693-709.

Goubert C., Minard G., Vieira C. \& Boulesteix M. (2016). Population genetics of the Asian tiger mosquito Aedes albopictus, an invasive vector of human diseases. Heredity 117(3): 125-134.

DOI: https://doi.org/10.1038/hdy.2016.35

Goudet J. (1995). Computer note computer program to calculate F-statistics. Journal of Heredity 86(6): 485-486.

Gunawickrama K.B.S. (2007). Morphological heterogeneity and population differentiation in the green chromid Etroplus suratensis (Pisces: Cichlidae) in Sri Lanka. Ruhuna Journal of Science 2: 70-81. 
Guo J., Wang Z. \& Francis F. (2017). Use of molecular markers for entomological diversity assessment and their application in population study of aphids. Entomologie faunistique - Faunistic Entomology 70: 49-62.

DOI: https://doi.org/10.25518/2030-6318.3647

Gupta S. \& Preet S. (2014). Genetic differentiation of invasive Aedes albopictus by RAPD-PCR: implications for effective vector control. Parasitology Research 113(6): 2137-2142. DOI: https://doi.org/10.1007/s00436-014-3864-2

Hall T. (1999). BioEdit: a user-friendly biological sequence alignment editor and analysis program for Windows 95/98/ NT. Nucleic Acids Symposium Series 41: 95-98. DOI: https://doi.org/citeulike-article-id:691774

Hlaing T. et al.(13 authors) (2010). Spatial genetic structure of Aedes aegypti mosquitoes in mainland Southeast Asia. Evolutionary Applications 3(4): 319-39. DOI: https://doi.org/10.1111/j.1752-4571.2009.00113.x

Hurst G.D.D. \& Jiggins F.M. (2005). Problems with mitochondrial DNA as a marker in population, phylogeographic and phylogenetic studies: The effects of inherited symbionts. Proceedings of the Royal Society B: Biological Sciences 272(1572): 1525-1534.

DOI: https://doi.org/10.1098/rspb.2005.3056

Janderson J.M., Lai J.E., Dotson E.M., Cordon-Rosales C., Ponce C., Norris D.E. \& Beard C.B. (2002). Identification and characterization of microsatellite markers in the Chagas disease vector Triatoma dimidiata. Infection, Genetics and Evolution 1(3): 243-248.

DOI: https://doi.org/10.1038/jid.2014.371

Lessa E.P. (1992). Rapid surveying of DNA sequence variation in natural populations. Molecular Biology and Evolution 9(2): 323-330.

DOI:https://doi.org/10.1093/oxfordjournals.molbev. a040723

Librado P. \& Rozas J. (2009). DnaSP v5: a software for comprehensive analysis of DNA polymorphism data. Bioinformatics 25(11): 1451-1452.

Minard G., Tran F.H., Van V.T., Fournier C., Potier P., Roiz D., Mavingui P. \& Moro C.V. (2018). Shared larval rearing environment, sex, female size and genetic diversity shape Ae. albopictus bacterial microbiota. PLoS ONE 13(4): $1-16$. DOI: https://doi.org/10.1371/journal.pone.0194521

Motoki M.T. et al. (11 authors) (2019). Population genetics of Aedes albopictus (Diptera: Culicidae) in its native range in Lao People's Democratic Republic. Parasites and Vectors 12(477): 1-12.

DOI: https://doi.org/10.1186/s13071-019-3740-0

National plan of action for prevention and control of dengue fever 2005-2009 (2005). Epidemiology Unit, Ministry of Health, Colombo, Sri Lanka. Available at $h t t p: / / w w w . e p i d$. gov.lk. Accessed 13 December 2019.

Olanratmanee P., Kittayapong P., Chansang C., Hoffmann A. A., Weeks A.R. \& Endersby N.M. (2013). Population genetic structure of Aedes (Stegomyia) aegypti (L.) at a micro-spatial scale in Thailand: implications for a dengue suppression strategy. PLoS Neglected Tropical Diseases 7(1): e1913.
DOI: https://doi.org/10.1371/journal.pntd.0001913

Rajkumar G., Weerasena J., Fernando K. \& Liyanage A. (2011). Assessment of genetic diversity among Sri Lankan rice varieties by AFLP markers. Plant Genetic Resources: Characterisation and Utilisation 9(2): 224-228. DOI: https://doi.org/10.1017/S1479262111000256

Romans P., Seeley D.C., Kew Y. \& Gwadz R.W. (1991). Use of a restriction fragment length polymorphism (RFLP) as a genetic marker in crosses of Anopheles gambiae (Diptera: Culicidae): independent assortment of a diphenol oxidase RFLP and an esterase locus. Journal of Medical Entomology 28(1): 147-151.

DOI: https://doi.org/10.1093/jmedent/28.1.147

Sandamal S., Tennakoon A., Meng Q.L., Marambe B., Ratnasekera D., Melo A. \& Ge S. (2018). Population genetics and evolutionary history of the wild rice species Oryza rufipogon and O. nivara in Sri Lanka. Ecology and Evolution 8(23): 12056-12065.

DOI: https://doi.org/10.1002/ece3.4665

Singh S., Mishra V.K. \& Bhoi T.K. (2017). Insect molecular markers and its utility-a review. International Journal of Agriculture, Environment and Biotechnology 10(4): 469-479. DOI: https://doi.org/10.5958/2230-732x.2017.00058.4

Slade R.W., Moritz C., Heideman A. \& Hale P.T. (1993). Rapid assessment of single-copy nuclear DNA variation in diverse species. Molecular Ecology 2(6): 359-373. DOI: https://doi.org/10.1111/j.1365-294X.1993.tb00029.x

Slatkin M. \& Barton N.H. (1989). A comparison of three indirect methods for estimating average levels of gene flow. Evolution 43(7): 1349-1368.

Slotman M. A., Kelly N.B., Harrington L.C., Kitthawee S., Jones J.W., Scott T.W., Caccone A. \& Powell J.R. (2007). Polymorphic microsatellite markers for studies of Aedes aegypti (Diptera: Culicidae), the vector of dengue and yellow fever. Molecular Ecology Notes 7(1): 168-171. DOI: https://doi.org/10.1111/j.1471-8286.2006.01533.x

Ströher P.R., Li C. \& Pie M.R. (2013). Exon-primed introncrossing (EPIC) markers as a tool for ant phylogeography. Revista Brasileira de Entomologia 57(4): 427-430. DOI: https://doi.org/10.1590/S0085-56262013005000039

Tay W.T., Behere G.T., Heckel D.G., Lee S.F. \& Batterham P. (2008). Exon-primed intron-crossing (Rp EPIC) PCR markers of Helicoverpa armigera (Lepidoptera: Noctuidae). Bulletin of Entomological Research 98(5): 509-518.

Tchouassi D.P., Bastos A.D.S., Sole C.L., Diallo M., Lutomiah J., Mutisya J., Mulwa F., Borgemeister C., Sang R. \& Torto B. (2014). Population genetics of two key mosquito vectors of rift valley fever virus reveals new insights into the changing disease outbreak patterns in Kenya. PLoS Neglected Tropical Diseases 8(12). DOI: https://doi.org/10.1371/journal.pntd.0003364

Touriya A., Rami M., Cattaneo-Berrebi G., Ibanez C., Augros S., Boissin E., Dakkak A. \& Berrebi P. (2003). Primers for EPIC amplification of intron sequences for fish and other vertebrate population genetic studies. BioTechniques 35(4): 676-682. 
Twerdochlib A.L., Bona A.C.D., Leite S.S., Chitolina R.F., Westphal B. \& Navarro-Silva M.A. (2012). Genetic variability of a population of Aedes aegypti from Paraná, Brazil, using the mitochondrial ND4 gene. Revista Brasileira de Entomologia 56(2): 249-256.

DOI: https://doi.org/10.1590/S0085-56262012005000030

Van Oosterhout C., Hutchinson W. F., Wills D. P. M. \& Shipley P. (2004). MICRO-CHECKER: Software for identifying and correcting genotyping errors in microsatellite data. Molecular Ecology Notes 4(3), 535-538. DOI: https://doi.org/10.1111/j.1471-8286.2004.00684.x

Viard F., Franck P., Dubois M., Estoup A. \& Jarne P. (1998). Variation of microsatellite size homoplasy across electromorphs, loci, and populations in three invertebrate species. Journal of Molecular Evolution 47(1): 42-51.

Waldock J., Chandra N.L., Lelieveld J., Proestos Y., Michael E., Christophides G. \& Parham P.E. (2013). The role of environmental variables on Aedes albopictus biology and chikungunya epidemiology. Pathogens and Global Health 107(5): 224-241.

DOI: $10.1179 / 2047773213$ Y.0000000100.

Weeraratne T.C., Surendran S.N., Walton C. \& Karunaratne S.H.P.P. (2018). Genetic diversity and population structure of malaria vector mosquitoes Anopheles subpictus, Anopheles peditaeniatus, and Anopheles vagus in five districts of Sri Lanka. Malaria Journal 17(1): 1-8. DOI: https://doi.org/10.1186/s12936-018-2419-x

Wei Y., Wang J., Song Z., He Y., Zheng Z., Fan P., Yang D., Zhou G., Zhong D. \& Zheng X. (2019). Patterns of spatial genetic structures in Aedes albopictus (Diptera: Culicidae) populations in China. Parasites and Vectors 12(552). DOI: https://doi.org/10.1186/s13071-019-3801-4
White V.L., Endersby N.M., Chan J., Hoffmann A.A. \& Weeks A.R. (2015). Developing exon-primed intron-crossing (EPIC) markers for population genetic studies in three Aedes disease vectors. Insect Science 22(3): 409-423. DOI: https://doi.org/10.1111/1744-7917.12145

World Health Oraganization (WHO) (1999). Comprehensive Guidelines for Prevention and Control of Dengue and Dengue Haemorrhagic Fever. WHO Regional Publications 29. World Regional Office for South-East Asia, India.

World Health Oraganization (WHO) (2009). Dengue: Guidelines for Diagnosis, Treatment, Prevention and Control. Available at: https://www.who.int/neglected diseases/resources/9789241547871/en/

Yan G., Chadee D.D. \& Severson D.W. (1998). Evidence for genetic hitchhiking effect associated with insecticide resistance in Aedes aegypti. Genetics 148(2): 793-800.

Yan G., Romero-Severson J., Walton M., Chadee D.D. \& Severson D.W. (1999). Population genetics of the yellow fever mosquito in Trinidad: comparisons of amplified fragment length polymorphism (AFLP) and restriction fragment length polymorphism (RFLP) markers. Molecular Ecology 8(6): 951-963.

DOI: https://doi.org/10.1046/j.1365-294X.1999.00647.x

Yang M., Ma Y. \& Wu J. (2011). Mitochondrial genetic differentiation across populations of the malaria vector Anopheles lesteri from China (Diptera: Culicidae). Malaria Journal 10(1).

DOI: https://doi.org/10.1186/1475-2875-10-216

Yeap H.L. et al. (11 authors) (2011). Dynamics of the "popcorn" Wolbachia infection in outbred Aedes aegypti informs prospects for mosquito vector control. Genetics 187(2): 583-595.

DOI: https://doi.org/10.1534/genetics. 110.122390 